\title{
Chromatin coming into focus
}

\author{
This special issue of $N S M B$ examines the structural organization and function of chromatin and its dynamic regulation.
}

$\mathbf{T}$ - he new Hall of Human Origins at the American Museum of Natural History in New York brings together evidence illustrating the evolutionary changes in hominid form that may have so profoundly affected function. Before letting the skeletons out of the closet, the exhibit features a molecular biology corner, which poses a question to all that dare enter: "If human and chimp DNA is 98.8\% the same, why are we so different?" An associated movie zooms in to a rod-cell nucleus, then to a chromosome, which unravels to briefly reveal arrayed nucleosomes packaging DNA before the final closeup of the DNA double helix itself.

It has become increasingly clear that, rather than simply representing packaged DNA, chromatin also has an ever-changing form with functional consequences most profound. Indeed, not only must $>2 \mathrm{~m}$ of DNA be organized and packed into the human nucleus, but this DNA is packaged into nucleosomes that are dynamically modified, shunted, removed and swapped out-and, to top it all off, undergo massive reorganization for mitosis, repair and other processes. Returning to the notion of stasis, however, some changes can be preserved and transmitted over cell cycles, and indeed over generations. These properties must reflect a remarkably malleable but stable chromatin structure at many levels, and in September 1997, researchers obtained a high-resolution glimpse of the basic unit underlying this structure, the nucleosome ${ }^{1}$. Reexamining the nucleosome structure illuminates both the questions that preceded its solution and those that have arisen in the ten years since. This Chromatin special focus issue of Nature Structural \& Molecular Biology (http://www. nature.com/nsmb/focus/chromatin) now brings together six Reviews and one Perspective that look at a range of current topics in the study of chromatin's structure, function and dynamic regulation.

The many inter-histone and DNA-histone contacts in the nucleosome, the latter of which partially occlude the DNA, raise the questions of how this structure is assembled and disassembled and how DNA is accessed during replication, repair, transcription and other processes. The histone chaperones form a class of proteins that can facilitate these processes and are considered by Almouzni and colleagues on page 997. Once assembled, the nucleosomes are not always a static set of regularly spaced 'beads on a string', but rather are actively altered by remodeling complexes. Although the molecular mechanisms underlying remodeling seem far from settled, a number of recent single-molecule observations have revealed unanticipated events, and in a Perspective on page 989, Cairns summarizes new insights from such approaches.

Structures of the nucleosome core particle strikingly illustrate the exposure of the $\mathrm{N}$-terminal histone tails, with the tail of histone $\mathrm{H} 3$ projecting far from the core nucleosomal disc itself. These tails house residues that are covalently modified, and such modifications are dynamic, have functional consequences and are thought to convey epigenetic information. Functions associated with covalent modifications, and the modifiers that regulate them, are discussed in the context of development and disease by Shilatifard and colleagues (page 1008). As it is increasingly clear that the complex array of histone tail modifications affect one another, Latham and Dent discuss the nuts and bolts of such cross-talk between modifications and its implications (page 1017). Covalent modifications have been proposed to represent a 'histone code', in some ways analogous to the genetic code, and on page 1025, Allis and Patel review how these changes are recognized and read by the cellular machinery.

The next Reviews consider how the structure of chromatin is organized and how functional territories are maintained at higherorder structural levels. Structural changes are key to setting up functional territories, and on page 1041, Bühler and Moazed discuss this in the context of heterochromatic regions. Whereas heterochromatin was once considered a transcriptional desert, they discuss how the genome sometimes 'runs to stand still', focusing on the paradox that heterochromatic silencing involves transcriptional activity in some species. Gasser, Fraser and colleagues then delve into structure-function relationships by examining chromatin organization within the nucleus at large, an area where molecular mechanisms are currently being elucidated (page 1049).

Given the complexity of this field and its pace of advance, the focus also includes primers that cover various subjects of research. These primers (page 1110) visually link structural insights to functional information in four areas: covalent modifications, histone-modification enzymes, histone recognition and, finally, chromatin-remodeling complexes. The versions of the primers on our website (http://www. nature.com/nsmb) let you view representatives of known highresolution structures in Jmol, allowing you to rotate and examine them in three dimensions without additional software.

Altogether, the focus issue presents not only the physically dynamic aspects of chromatin structure, but also the changing face of a field that continues to yield surprises. The research reviewed here reveals the profound impact of this packaging on the genome that it packagesand the interplay of the two. Indeed, studies in this area may ultimately form part of the answer to that question: why are we so different?

We would like to thank all of the experts in the field who wrote, advised, commented and peer-reviewed, for their hard work and insightful suggestions and input. We hope you enjoy reading this collection of Reviews, and we now end with the beginning, handing you over to Roger Kornberg for a historical overview of the field that brings us up to the present and leaves us with a sense of anticipation of what the next decade will reveal.

1. Luger, K., Mäder, A.W., Richmond, R.K., Sargent, D.F. \& Richmond, T.J. Crystal structure of the nucleosome core particle at $2.8 \AA$ resolution. Nature $389251-260$ (1997). 